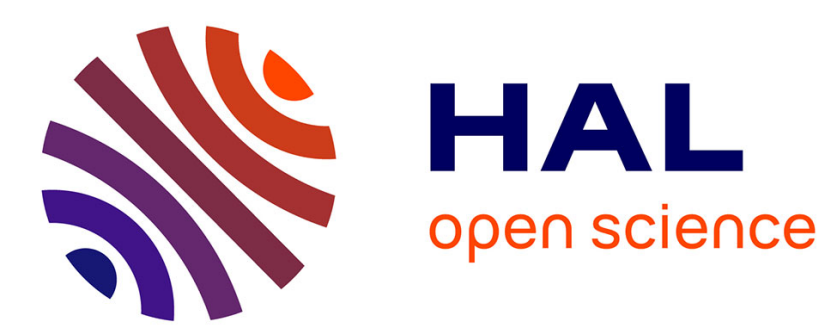

\title{
L'ion hydrogénoïde piégé en électrodynamique quantique non relativiste
}

Laurent Amour, Jérémy Faupin

\section{To cite this version:}

Laurent Amour, Jérémy Faupin. L'ion hydrogénoïde piégé en électrodynamique quantique non relativiste. Comptes rendus de l'Académie des sciences. Série I, Mathématique, 2006, 343, pp.27-30. hal-00179721

\section{HAL Id: hal-00179721 \\ https://hal.science/hal-00179721}

Submitted on 16 Oct 2007

HAL is a multi-disciplinary open access archive for the deposit and dissemination of scientific research documents, whether they are published or not. The documents may come from teaching and research institutions in France or abroad, or from public or private research centers.
L'archive ouverte pluridisciplinaire HAL, est destinée au dépôt et à la diffusion de documents scientifiques de niveau recherche, publiés ou non, émanant des établissements d'enseignement et de recherche français ou étrangers, des laboratoires publics ou privés. 


\title{
L’ion hydrogénoïde piégé en électrodynamique quantique non relativiste
}

\author{
Laurent AMOUR, Jérémy FAUPIN \\ Laboratoire de Mathématiques, UMR-CNRS 6056, Université de Reims, \\ Moulin de la Housse - BP 1039, 51687 REIMS Cedex 2, France. \\ laurent.amour@univ-reims.fr, jeremy.faupin@univ-reims.fr
}

\section{Résumé}

Nous considérons un noyau et un électron non relativistes interagissant avec un champ électromagnétique quantifié. Nous ne supposons pas le noyau fixe, mais nous supposons que le système est confiné par son centre de masse. Ce modèle est utilisé en physique théorique pour décrire l'effet

Lambe-Dicke et l'effet Mössbauer. Nous définissons l'hamiltonien associé au système en introduisant une troncature ultraviolette, puis nous prouvons l'existence d'un état fondamental non dégénéré. Ce résultat est obtenu sans condition sur les constantes de couplage.

\section{The confined hydrogenoïd ion in non-relativistic quantum electrodynamics.}

Abstract

We consider a system of a nucleus with an electron together with the quantized electromagnetic field. Instead of fixing the nucleus, the system is confined by its center of mass. This model is used in theoretical physics to explain the Lamb-Dicke and the Mössbauer effects. When an ultraviolet cut-off is imposed we initiate the spectral analysis of the Hamiltonian describing the system and we derive the existence of a ground state. This is achieved without conditions on the fine structure constant.

\section{Définition du modèle}

Nous considérons un noyau (assimilé à une particule) de masse $m_{1}$ et de charge $q_{1}$, interagissant par l'intermédiaire du potentiel de Coulomb, noté $V$, avec un électron de masse $m_{2}$ et de charge $q_{2}$. Nous supposons également que les deux particules interagissent avec le champ électromagnétique quantifié en jauge de Coulomb. Le mouvement du centre de masse de l'ion est supposé confiné par un potentiel $U$. Le fait que le noyau soit mobile, et non pas fixe, modifie de manière significative le spectre de diffusion du système. Ce modèle est en particulier utilisé pour expliquer l'effet LambDicke, ou encore l'effet Mössbauer (cf. [CTDRG]). Mentionnons par ailleurs un autre cas de système ayant un noyau non fixe, étudié dans [AGG] : le cas des atomes et des ions mobiles en interaction avec un champ électromagnétique quantifié.

Pour plus de simplicité, nous considérons que le spin des deux particules est nul, et que $\hbar=c=1$. 
L'espace de Hilbert associé à ce système est alors

$$
\mathcal{H}:=\mathrm{L}^{2}\left(\mathbb{R}^{6}\right) \otimes \mathcal{F}_{s}:=\mathrm{L}^{2}\left(\mathbb{R}^{6}\right) \otimes \bigoplus_{n=0}^{\infty} \mathrm{L}^{2}\left(\mathbb{R}^{3} ; \mathbb{C}^{2}\right)^{\otimes_{s}^{n}}
$$

où $\mathcal{F}_{s}$ est l'espace de Fock symétrique associé aux photons. Nous notons $R, P$ les variables associées au centre de masse, définies par

$$
R:=\frac{m_{1} x_{1}+m_{2} x_{2}}{m_{1}+m_{2}} \quad, \quad P:=p_{1}+p_{2}
$$

et $r, p$ les variables internes, définies par

$$
r:=x_{1}-x_{2}, \quad, \quad p:=\frac{m_{2}}{m_{1}+m_{2}} p_{1}-\frac{m_{1}}{m_{1}+m_{2}} p_{2} .
$$

L'hamiltonien de Pauli-Fierz dans $\mathcal{H} \simeq \mathrm{L}^{2}\left(\mathbb{R}^{6} ; \mathcal{F}_{s}\right)$ est formellement donné par

$$
H_{U}^{V}:=\sum_{j=1,2} \frac{1}{2 m_{j}}\left(p_{j}-q_{j} A_{j}\right)^{2}+H_{f}+U+V
$$

où $p_{j}=-i \nabla_{j}$ est l'impulsion de la particule $j, A_{j}=\int_{\mathbb{R}^{6}}^{\oplus} A\left(x_{j}\right) d X$, avec $X=\left(x_{1}, x_{2}\right)$, est le champ électromagnétique quantififé en jauge de Coulomb, $H_{f}$ est l'hamiltonien du champ de photons, $U$ et $V$ sont des fonctions de $R$ et $r$ respectivement. Formellement, $A(x)$ et $H_{f}$ sont donnés respectivement par :

$$
\begin{aligned}
A(x) & =\frac{1}{2 \pi} \sum_{\lambda=1,2} \int_{\mathbb{R}^{3}} \frac{\widehat{\chi}_{\Lambda}(k)}{|k|^{1 / 2}} \varepsilon_{\lambda}(k)\left(\widehat{a}_{\lambda}^{*}(k) e^{-i k \cdot x}+\widehat{a}_{\lambda}(k) e^{i k \cdot x}\right) d k, \\
H_{f} & =\sum_{\lambda=1,2} \int_{\mathbb{R}^{3}}|k| \widehat{a}_{\lambda}^{*}(k) \widehat{a}_{\lambda}(k) d k .
\end{aligned}
$$

Nous choisissons la fonction de troncature ultraviolette $\widehat{\chi}_{\Lambda}$ dans $\mathrm{C}_{0}^{\infty}\left(\mathbb{R}^{3}\right)$, positive et nulle en dehors de $B(0, \Lambda)$, où $\Lambda$ est le paramètre ultraviolet. De plus, nous choisissons les vecteurs de polarisation suivants :

$$
\varepsilon_{1}(k):=\frac{\left(k_{2},-k_{1}, 0\right)}{\sqrt{k_{1}^{2}+k_{2}^{2}}} \quad, \quad \varepsilon_{2}(k):=\frac{k}{|k|} \wedge \varepsilon_{1}(k) .
$$

Enfin, $\widehat{a}_{\lambda}^{*}(k)$ et $\widehat{a}_{\lambda}(k)$ sont les vecteurs de création et d'annihilation habituels satisfaisant aux relations canoniques de commutation :

$$
\left[\widehat{a}_{\lambda}^{*}(k), \widehat{a}_{\lambda^{\prime}}^{*}\left(k^{\prime}\right)\right]=\left[\widehat{a}_{\lambda}(k), \widehat{a}_{\lambda^{\prime}}\left(k^{\prime}\right)\right]=0 \quad, \quad\left[\widehat{a}_{\lambda}(k), \widehat{a}_{\lambda^{\prime}}^{*}\left(k^{\prime}\right)\right]=\delta_{\lambda \lambda^{\prime}} \delta\left(k-k^{\prime}\right) .
$$

Pour les besoins de la démonstration, nous supposons que $U$ vérifie les hypothèses suivantes :

1. $U \in \mathrm{L}_{\text {loc }}^{1}\left(\mathbb{R}^{3}\right)$,

2. $\inf U>-\infty$ et $U^{-}$est support compact (où $U^{-}$désigne la partie négative de $U$ ),

3. $P^{2} / 2 M+U$ a un état fondamental non dégénéré $\phi>0$ d'énergie $-e_{0}<0$, et il existe $\gamma>0$ tel que $|\phi(R)| \leq \gamma e^{-|R| / \gamma}$. 
Pour $m>0$, nous définissons également l'hamiltonien massif $H_{U}^{V}(m)$ de la même façon que $H_{U}^{V}$, à ceci près que $H_{f}$ est remplacé par $H_{f}(m)$, où :

$$
H_{f}(m)=\sum_{\lambda=1,2} \int_{\mathbb{R}^{3}} \sqrt{k^{2}+m^{2}} \widehat{a}_{\lambda}^{*}(k) \widehat{a}_{\lambda}(k) d k
$$

et nous posons $H_{U}^{V}(0)=H_{U}^{V}$.

\section{Résultats et commentaires}

Nous montrons tout d'abord en utilisant les formes quadratiques que $H_{U}^{V}(m)$ (avec $m \geq 0$ ) est correctement défini :

Théorème 2.1 L'opérateur $H_{U}^{V}(m), m \geq 0$, est auto-adjoint dans $\mathcal{H}$, semi-borné inférieurement, et le domaine de la forme quadratique qui lui est associée est $Q\left(H_{U}^{V}(m)\right)=Q\left(p_{1}^{2}+p_{2}^{2}\right) \cap Q\left(H_{f}(m)\right) \cap$ $Q\left(U^{+}\right)$.

\section{Remarque 2.1}

- Nous déduisons facilement de ce résultat un corollaire largement utilisé dans la suite : si $m>0$, $Q\left(H_{U}^{V}(m)\right)=Q\left(H_{U}^{V}\right) \cap Q(\mathcal{N})$, où $\mathcal{N}:=\sum_{\lambda} \int \widehat{a}_{\lambda}^{*}(k) \widehat{a}_{\lambda}(k) d k$ est l'opérateur du nombre de photons.

- Une autre méthode pour définir l'hamiltonien associé au système repose sur la représentation de Schrödinger de l'espace de Fock, et est présentée dans [H1]. Plus précisément, grâce à une représentation en intégrale fonctionnelle de $e^{-t H_{0}^{0}}$, il est montré que l'opérateur $H_{U-}^{V}$ est autoadjoint sur $D\left(p_{1}^{2}+p_{2}^{2}\right) \cap D\left(H_{f}\right)$. Il suffit alors de définir $H_{U}^{V}=H_{U^{-}}^{V}+U^{+}, \dot{+}$ désignant la somme au sens des formes quadratiques.

Nous prouvons que les deux définitions, celle de [H1] et la nôtre, coïncident. L'avantage de notre définition est de connaître explicitement le domaine $Q\left(H_{U}^{V}\right)$ que nous utilisons à de nombreuses reprises au cours de la preuve.

Nous nous intéressons ensuite à l'existence d'un état fondamental pour $H_{U}^{V}$. Nous basant sur la stratégie de [G], [GLL] et [LL], la clé de notre démonstration est de choisir correctement des conditions de liaison, de manière à ce que, d'une part, nous puissions les vérifier, et que d'autre part, elles entraînent l'existence d'un état fondamental. Les conditions que nous avons choisies sont les suivantes :

$$
\begin{aligned}
\text { (i) } & E\left(H_{U}^{V}\right)<E\left(H_{0}^{V}\right), \\
\text { (ii) } & E\left(H_{U}^{V}\right)<E\left(H_{U}^{0}\right),
\end{aligned}
$$

où $E\left(H_{U}^{V}\right)$ est l'infimum du spectre de $H_{U}^{V}$ et où $H_{0}^{V}$ (respectivement $H_{U}^{0}$ ) désigne $H_{U}^{V}$ où l'on a fait $U=0$ (respectivement $V=0$ ). Nous montrons alors :

Théorème 2.2 Les conditions $(i)$ et (ii) sont vérifiées. Ceci implique que $H_{U}^{V}$ possède un état fondamental non dégénéré, quelles que soient les valeurs de $q_{1}, q_{2}$ et $\Lambda$. 


\section{Remarque 2.2}

- Pour prouver l'existence d'un état fondamental lorsque (i) et (ii) sont supposées valides, nous suivons la méthode de [GLL]. Quelques modifications sont toutefois nécessaires dans la mesure où les conditions de liaison que nous avons choisies sont différentes de celles de [GLL]. En particulier, nous obtenons la décroissance exponentielle de l'état fondamental pour l'hamiltonien massif sans avoir recours à la méthode développée dans $[\mathrm{G}]$.

Par ailleurs, la non dégénérescence est une conséquence de la représentation en intégrale fonctionnelle mentionnée à la remarque 2.1. (cf. [H2]).

- La démonstration de la condition (i) suit également [GLL]. Ceci est possible notamment en raison de l'invariance par translation de $H_{0}^{V}$.

- En revanche, $H_{U}^{0}$ n'est pas invariant par translation, si bien que la démonstration de la condition (ii) s'avère plus difficile. Ce point constitue notre principal résultat. Nous nous sommes appuyés pour l'obtenir sur les méthodes développées dans [LL], qui permettent de localiser à la fois noyau, électron et photons. Plus précisément, considérant une suite normalisée $\left(F_{j}\right)$, minimisante pour $H_{U}^{0}$, nous montrons :

\section{Proposition 2.1}

S'il existe $\rho>0$ et $a>0$ tels que $\forall j, \int_{B(0, \rho)} \int_{\mathbb{R}^{3}}\left\|F_{j}(X)\right\|^{2} d R d r \geq a$, alors

$$
E\left(H_{U}^{V}\right) \leq E\left(H_{U}^{0}\right)-\mathrm{C} a / \rho,
$$

où $\mathrm{C}$ est la constante définie par $V(r)=-\mathrm{C} /|r|$.

Si au contraire, $\forall n \in \mathbb{N}^{*}, \exists j_{n}, \int_{B(0, n)} \int_{\mathbb{R}^{3}}\left\|F_{j_{n}}(X)\right\|^{2} d R d r \leq \frac{1}{n}$, alors

$$
E\left(H_{U}^{V}\right)<E\left(\widetilde{H}_{U}^{0}\right) \leq E\left(H_{U}^{0}\right),
$$

où nous avons défini l'opérateur $\widetilde{H}_{U}^{0}$ dans $\mathrm{L}^{2}\left(\mathbb{R}^{6}\right) \otimes \mathcal{F}_{s} \otimes \mathcal{F}_{s}$ de la façon suivante :

$$
\widetilde{H}_{U}^{0}:=\frac{1}{2 m_{1}}\left(p_{1}-q_{1} A_{1}\right)^{2} \otimes I+\frac{1}{2 m_{2}} I \otimes\left(p_{2}-q_{2} A_{2}\right)^{2}+H_{f} \otimes I+I \otimes H_{f}+U .
$$

\section{Esquisse de démonstration}

La difficulté de la preuve réside dans les inégalités (11), la vérification de (10) étant immédiate. Pour montrer $E\left(\widetilde{H}_{U}^{0}\right) \leq E\left(H_{U}^{0}\right)$, l'idée est de partir de l'état $F_{j_{n}}$, puis de localiser chacune des deux particules (noyau et électron) dans une boule de rayon fixé. Nous localisons ensuite les photons autour des deux boules précédentes. Alors, comme lorsque $n$ est grand, les deux particules sont "loin" l'une de l'autre dans l'état $F_{j_{n}}$, les deux boules obtenues (où sont localisées chacune des deux particules entourée de son nuage de photons propre) sont également loin l'une de l'autre. Ceci permet de créer un état dans $\mathrm{L}^{2}\left(\mathbb{R}^{6}\right) \otimes \mathcal{F}_{s} \otimes \mathcal{F}_{s}$ dont l'énergie pour $\widetilde{H}_{U}^{0}$ est plus petite que $E\left(H_{U}^{0}\right)+\varepsilon$, où $\varepsilon$ dépend de $n$ et du rayon des boules de localisation, mais peut être rendu aussi petit qu'on le veut. 
Nous prouvons l'autre inégalité $E\left(H_{U}^{V}\right)<E\left(\widetilde{H}_{U}^{0}\right)$ selon une méthode similaire, en utilisant préalablement le fait que $\widetilde{H}_{U}^{0}$ est invariant par les translations conjointes de $x_{1}$ et $x_{2}$ qui ne modifient pas la position du centre de masse. Plus précisément, en posant

$$
\mathcal{U}_{t}=e^{i t \frac{m_{2}}{M}\left(p_{1}+d \Gamma(k)\right)} \otimes e^{-i t \frac{m_{1}}{M}\left(p_{2}+d \Gamma(k)\right)},
$$

nous pouvons remarquer que $\mathcal{U}_{t}^{*} \widetilde{H}_{U}^{0} \mathcal{U}_{t}=\widetilde{H}_{U}^{0}$. C'est là une caractéristique essentielle de $\widetilde{H}_{U}^{0}$, sans laquelle on ne pourrait conclure. Finalement, nous obtenons une inégalité stricte, en prouvant que les erreurs dues à la localisation des particules sont négligeables par rapport à l'énergie apportée par $V$.

\section{References}

[AGG] L. Amour, B. Grébert and J. C. Guillot. The dressed mobile atoms and ions. Journal de Mathématiques Pures et Appliquées, to appear.

[CTDRG] C. Cohen-Tannoudji, J. Dupont-Roc and G. Grynberg. Processus d'interaction entre photons et atomes. Edition du CNRS, 2001.

[G] M. Griesemer. Exponential decay and ionization thresholds in non-relativistic quantum electrodynamics. arXiv:math-ph/0206024, 2002.

[GLL] M. Griesemer, E. H. Lieb and M. Loss. Ground states in non-relativistic quantum electrodynamics. Invent. Math. 145:557-595, 2001.

[H1] F. Hiroshima. Self-adjointness of the Pauli-Fierz Hamiltonian for arbitrary coupling constants. Ann. Henri Poincaré 3:171-201, 2002.

[H2] F. Hiroshima. Ground states of a model in nonrelativistic quantum electrodynamics. II. J. math. phys. 41:661-674, 2000.

[LL] E. Lieb and M. Loss. Existence of atoms and molecules in non-relativstic quantum electrodynamics. Adv. Theor. Math. Phys., 7:667-710, 2003. 